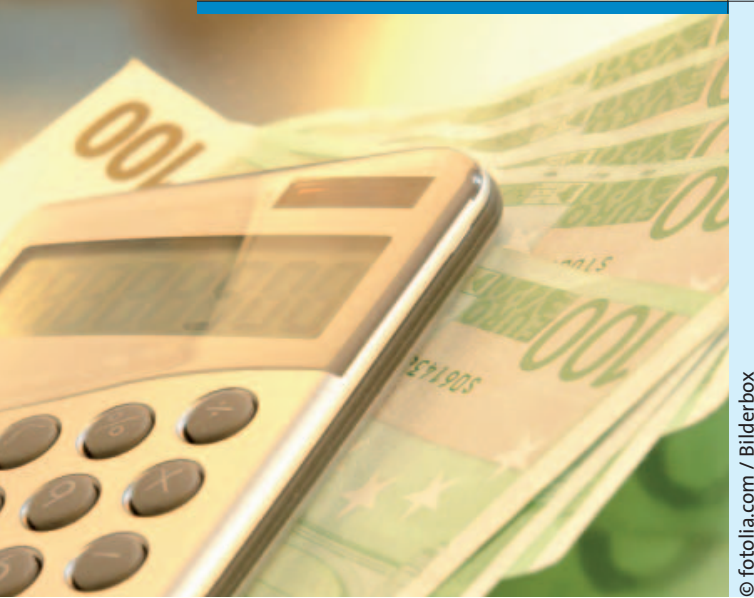

Fragen zur GOÄ-Abrechnung
Kostenloser Leserservice!

Haben Sie Fragen zur privatärztlichen Abrechnung? Antworten gibt unser Experte Dr. med.

Helmut Hoffmann, München.

Schreiben Sie an:

Urban \& Vogel GmbH

Redaktion HNO-NACHRICHTEN

Neumarkter Str. 43

81673 München

Fax 08943 72-14 00

E-Mail: sebastian.lux@springer.com

\title{
Wie kann eine Conchotomie korrekt abgerechnet werden?
}

Antwort: Bei der GOÄ-Nr. 1438 (teilweise oder vollständige Abtragung einer Nasenmuschel; 370 Punkte) handelt es sich zweifelsfrei um eine selbstständige Zielleistung mit eigener Indikation und nicht um eine Teilleistung etwa zur Erweiterung des Zugangsweges bei anderen Operationen. Die Nasenmuschelhyperplasie oder -hypertrophie ist ein eigenständiges Krankheitsbild neben einer eventuellen Septumdeviation und/ oder einer chronischen Nasennebenhöhlenentzündung. Die Nr. 1438 hat als Zielleistung, die Größe beziehungsweise das Volumen einer Nasenmuschel zu reduzieren.

Die konventionelle teilweise oder vollständige Abtragung einer Nasenmuschel ist gemäß den Beschlüssen des Zentralen
Konsultationsausschusses 2004 GOÄ-Nr. 1438 zuzuordnen. Operationen an den knöchernen Anteilen der Nasenmuscheln können nicht als selbstständige Osteotomie nach Nr. 2250 neben der Nr. 1438 berechnet werden.

Eine schleimhautschonende plastische Operation an der Nasenmuschel (z.B. Turbinoplastik) oder der Eingriff nach Legler ist gemäß den Beschlüssen des Zentralen Konsultationsausschusses 2004 analog nach GOÄ-Nr. 2382 zu bewerten. Damit sind alle an dieser Nasenmuschel erforderlichen Maßnahmen an Schleimhaut, Weichteilen und gegebenenfalls knöchernen Anteilen der Nasenmuschel abgegolten. Eine zusätzliche Berechnung der GOÄ-Nr. 1430 für dieselbe Nasenmuschel ist somit nicht korrekt.
Wenn Sie neben der Conchotomie auch die hinteren Siebbeine und/oder die Keilbeinhöhlen operiert haben und dafür GOÄ-Nr. 1470 (Keilbeinhöhlenoperation oder Ausräumung der Siebbeinzellen von der Nase aus - einschließlich teilweiser oder vollständiger Abtragung einer Nasenmuschel oder von Auswüchsen der Nasenscheidewand; 739 Punkte) in Rechnung stellen, ist zu beachten, dass diese Gebührenordnungsposition obligat die teilweise oder vollständige Abtragung einer Nasenmuschel beinhaltet. Eventuelle dadurch hervorgerufene Doppelberechnungen der Conchotomie werden von privaten Versicherungsgesellschaften immer häufiger zurecht moniert und nicht erstattet.

\section{Welche GOÄ-Ziffer würden Sie zur Abrechnung für eine Zungengrundmandel-Reduktion empfehlen?}

\author{
Antwort: Zunächst kommt es darauf an, \\ wie umfangreich und mit welcher Me- \\ thode die Hyperplasie der Zungengrund- \\ tonsille operativ beseitigt werden soll. \\ Wird nur ein Keil aus der Zungengrund- \\ tonsille entfernt, hält die GOÄ dafür die \\ Nr. 513 (Keilexzision aus der Zunge; 370 \\ Punkte) vor. Nr. 513 umfasst auch den \\ Wundverschluss. \\ Wird hingegen eine sehr umfangreiche \\ operative Verkleinerung der Zunge (even- \\ tuell mit Unterbindung der Arteria lingu- \\ alis) vorgenommen, was eigentlich nur \\ wegen eines Tumorleidens und damit \\ nur einseitig notwendig werden würde, \\ kann dafür Nr. 1512 (teilweise Entfernung \\ der Zunge gegebenenfalls einschließlich \\ Unterbindung der Arteria lingualis; 1.110 \\ Punkte) in Rechnung gestellt werden. \\ Wenn die Reduktion der Zungengrund- \\ tonsille lasergestützt oder mit der \\ Radiofrequenztherapie durchgeführt
}

wird, kann für diese Leistung GOÄ-Nr. 2404 (analog Exzision einer größeren Geschwulst - z.B. Ganglion, Fasziengeschwulst, Fettgeschwulst, Lymphdrüse, Neurom; 554 Punkte) in Rechnung gestellt werden.

Entscheidend sind somit die Art und Ausdehnung des Eingriffs sowie die entsprechende Dokumentation der Operationsschritte im OP-Bericht. 